\title{
Magnetic Polymer Nanocomposite for Medical Application
}

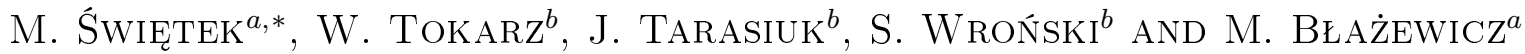 \\ ${ }^{a}$ Department of Biomaterials, Faculty of Materials Science and Ceramics, AGH University of Science and Technology \\ Al. A. Mickiewicza 30, 30-059 Krakow, Poland \\ ${ }^{b}$ Faculty of Physics and Applied Computer Science, AGH University of Science and Technology \\ Al. A. Mickiewicza 30, 30-059 Krakow, Poland

\begin{abstract}
Magnetic sponges derived from biocompatible and resorbable polymers are promising materials for medical applications. These materials have been utilised extensively in research applications for the capture of biomolecules and cells, the construction of tissue scaffolds and in regenerative medicine. The object of this study was a polymer scaffold made of polycaprolactone (PCL) containing a $10 \mathrm{wt} \%$ amount of nanomagnetite, manufactured in a two-step method. The porosity and morphological parameters were characterised with the use of $\mu$-computer-aided tomography and scanning electron microscopy. Furthermore, the magnetic properties were evaluated. The obtained results confirmed high porosity and the appearance of randomly oriented pores. Moreover, evaluations of the magnetic properties, of both the magnetite nanopowder and the prepared magnetic nanocomposite, were performed. The tests verified the ferromagnetic character of the materials under investigation.
\end{abstract}

DOI: $10.12693 /$ APhysPolA.125.891

PACS: 82.35.Np, 87.85.-d, 87.85.Rs, 75.75.-c, 87.85.J-, 87.85.Lf

\section{Introduction}

Currently, three-dimensional (3D) porous polymer materials are very popular due to their various potential applications in tissue engineering and regenerative medicine $[1,2]$. Owing to the ease in controlling the shape of their pores and the pores' size distribution, 3D porous materials constitute perfect scaffolds for cell cultures, giving optimal conditions for cell proliferation and differentiation, both in vitro and in vivo terms.

Additionally, the area of potential applications increases considerably with the possibility of incorporating various fillers into the polymer matrix such as: hydroxyapatite (HAp) [3, 4], $\beta$-tricalcium phosphate $(\beta$-TCP) [5-7], bio-glass [8-10] and magnetic particles [1]. The addition of the above-mentioned dopes greatly influences the mechanical, physicochemical and bioactive properties of the fabricated materials.

In particular, magnetic nanoadditives possess very promising characteristics in the field of polymer modification. Magnetic nanoparticles are widely used in medicine e.g. in cancer therapy (especially in the hyperthermia method), MRI diagnosis and drug targeting [11-15]. In addition, polymer foam containing magnetite nanoparticles can serve as a kind of receiver for biological agents (drugs) associated with magnetic particles (implant assisted magnetic drug targeting), or as tissue scaffold for biological modification in a way not possible to achieve in other materials [1]. This approach enables the long-term drug delivery process and supports tissue regeneration to be controlled adequately to requirement, even for a long period of time after implantation. Additionally,

*corresponding author; e-mail: swietek@agh.edu.pl magnetic implants, due to presence of particles possessing magnetic properties, also enhance and accelerate cell proliferation and bone tissue formation under an applied static magnetic field $[16,17]$.

The aim of our research is to obtain porous nanocomposite foam from a bioresorbable and biocompatible polymer, modified with magnetite nanoparticles. Magnetite nanoparticles are biocompatible and do not constitute a risk to the human body.

There are many known methods of polymer foam production for applications in technology and medicine $[18,19]$. However, the generation of polymer foam that contains magnetic nanoparticles is not an easy task due to a key issue, i.e. the appropriate dispersion of nanoparticles throughout the foam. Nanoparticles, especially magnetic ones, exhibit a very high tendency towards agglomeration. 3D porous materials functionalised with magnetic particles are generally obtained by way of immersion in a ferromagnetic liquid [1] with the use of co-precipitation methods [20], or using a variety of surfactants applied to the surface of the nanoparticles to prevent their agglomeration.

\section{Experimental}

\subsection{Materials}

Iron(II,II) oxide (magnetite) nanopowder of grain size below $50 \mathrm{~nm}$, and polycaprolactone (PCL) of average molar masses $\left(M_{n}\right)$ of 45000 , were purchased from Sigma Aldrich Co. Dichloromethane was obtained from POCH. Sodium chloride $(\mathrm{NaCl})$ was sieved using an ultrasonic sieving processor in order to obtain two grain size fractions of 200 and $300 \mu \mathrm{m}$ that served as a porogen.

\subsection{Methods}

2.2.1. Fabrication of PCL/magnetite scaffold

For the preparation of porous foam - PCL/magnetite - a modified version of the salt leaching method was ap- 
plied. Salt leaching is a commonly used method for polymer sponge fabrication [21-23]. Application of the salt leaching method makes it possible to produce magnetic polymer foam without the introduction of any additional chemical compounds in contrary to the above-mentioned methods e.g. co-precipitation or immersion in ferromagnetic liquid.

Polymer was first dissolved in dichloromethane, in order to obtain a $10 \% \mathrm{w} / \mathrm{w}$ polymer solution and homogenised continuously for $24 \mathrm{~h}$ on a magnetic stirrer. Both fractions of porogen and nanomagnetite powder $(10 \% \mathrm{w} / \mathrm{w}$ in relation to the mass of polymer) were mixed together. The blend was thoroughly shaken in order to provide an excellent component mixture. The salt fraction ratio was $1: 2(200 \mu \mathrm{m}: 300 \mu \mathrm{m})$. The polymer solution was mixed with a powder composition, put into a template, and left in order to evaporate the dissolvent. Afterwards, the porogen was leached.

\subsubsection{Morphology and composition characterization}

The morphological characteristics of the obtained materials were acquired with the use of a scanning electron microscope (SEM) equipped with an electron X-ray diffraction (EDS) attachment. The chemical composition analysis was performed for selected points during the microscopic observations. The porosity and the parameters of the structure were evaluated by means of X-ray computed tomography ( $\mu$-CAT), with the use of a Nanotom S scanner.

\subsubsection{Magnetic measurement}

For the magnetic properties characterisation, magnetic measurements of the PCL/magnetite sponge were performed. The measurements were carried out at room temperature $(300 \mathrm{~K})$ with a Lakeshore VSM magnetometer. The results of the magnetic properties of the polymer foam are presented as mean values of five independent measurements.

\section{Results}

Figure 1 represents the SEM micrographs of the obtained polymer sponge and their corresponding EDS analysis. The foam microstructure depicted in the SEM microscopy is characteristic for polymer materials produced by means of the salt leaching technique. SEM evaluation confirmed both the presence of randomly oriented pores and the presence of agglomerates of magnetic nanoparticles in the polymer sponge. Pore size and shape correspond to the respective properties of sodium chloride crystals which were used as the porogen. As shown in the microscopic images, the porosity of the foam is complex. Except the pores which have a shape similar to the shape of the porogen particles, much smaller pores are also visible on the walls of large pores.

EDS analysis confirms the presence of magnetic agglomerates of different sizes in a polymer sponge. Different intensity of the bands in EDS spectra, associated with the presence of iron, in the studied samples, indicate the uneven distribution of the magnetic particles and the different size of the agglomerates formed by them.
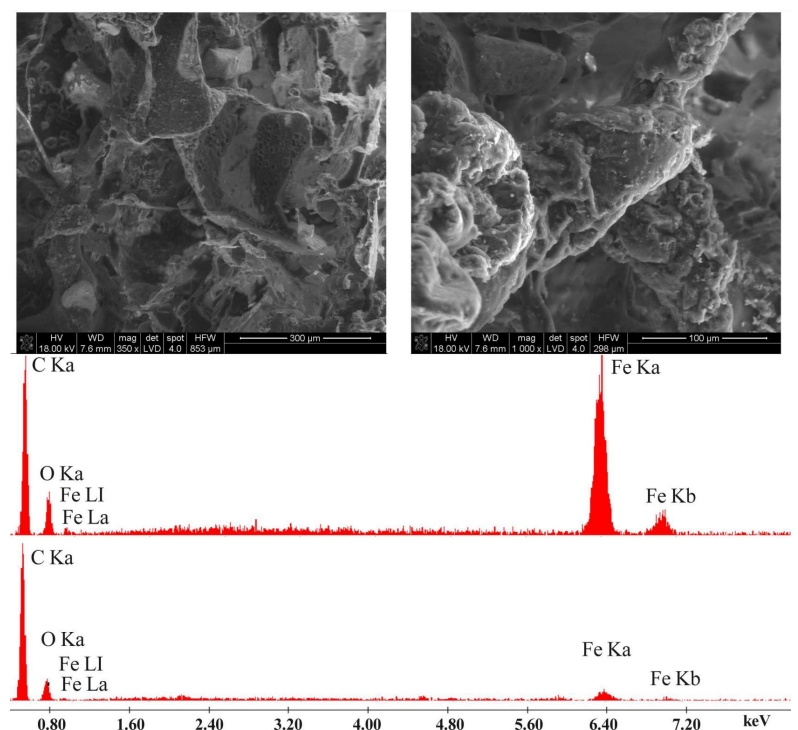

Fig. 1. SEM micrographs of fabricated polymer magnetic sponge and corresponding to them results of EDS analyses.

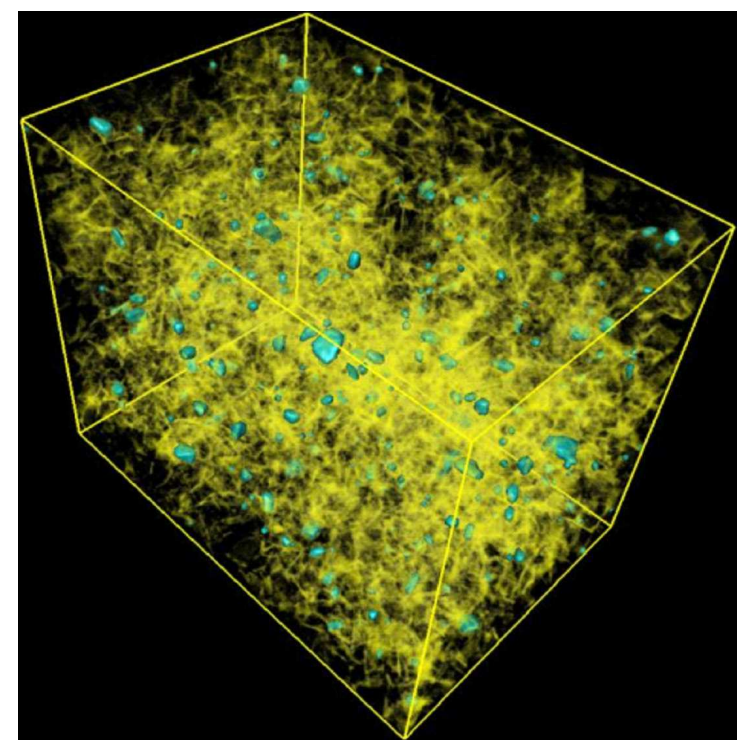

Fig. 2. CT visualization of polymer sponge (yellow areas) including magnetite interjections (blue region) in volume of the materials.

Figure 2 represents the visualisation of the magnetic sponge, achieved with the use of the $\mu$-CAT method. Figure 3 lays out the cross-section of the fabricated material. High-resolution $\mu$-CAT is used to analyse the array of microstructural parameters, which could be difficult to obtain by means of other methods, especially in the case of materials produced in the form of a sponge. The $\mu$-CAT results confirm the previous results concerning the magnetic sponge's structure and the appearance of a heterogenic size distribution of the magnetite agglomerates. 


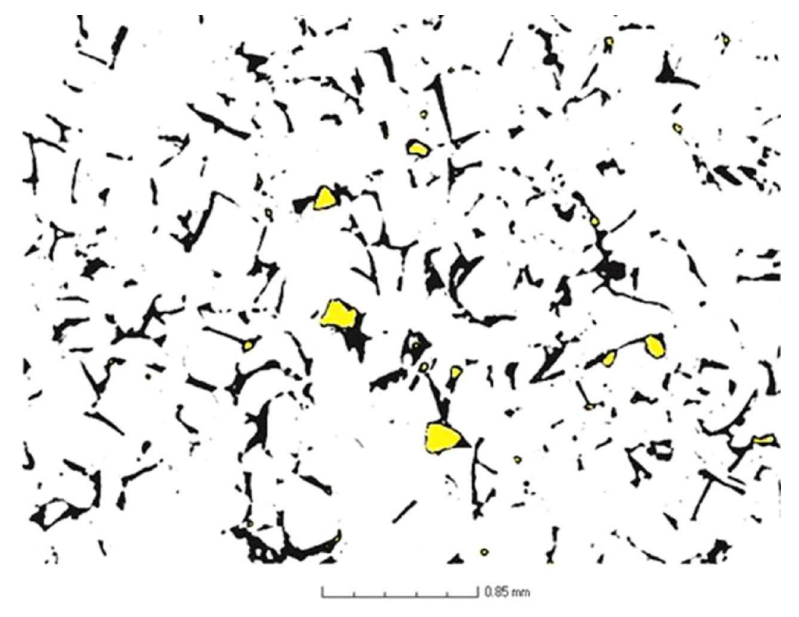

Fig. 3. Cross-section of polymer sponge with observable magnetite agglomerates (yellow districts).

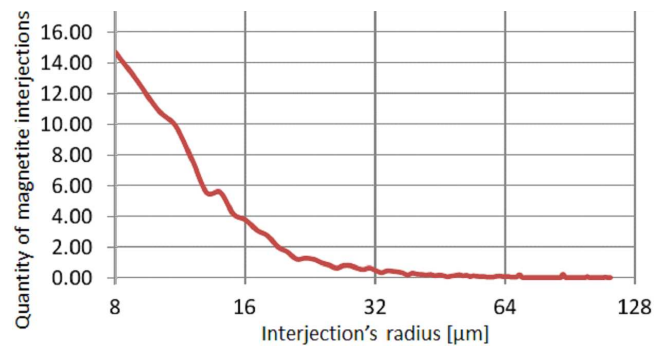

Fig. 4. Dependence between interjections' radius and their quantity.

The measurement-based accumulated curve, presented in Fig. 4, shows the dependence between the agglomerates' quantity and their size. It is proven that the amount of the large agglomerates in relation to small ones is low. The particle size is inversely proportional to their quantity. The evaluation method of particle size $(\mu-\mathrm{CAT})$ was performed at a resolution of $8 \mu \mathrm{m}$. However, it can be assumed that within the polymeric sponge there is a significant quantity of particles forming agglomerates with sizes much smaller than $8 \mu \mathrm{m}$. According to the previously presented study on the microstructure of the polymer sponge, this material is characterised by regu-

TABLE I

Magnetic sponge's parameters obtained from micro-CT method.

\begin{tabular}{c|c|c|c}
\hline \hline $\begin{array}{c}\text { Polymer volume } \\
{\left[\mathrm{mm}^{3}\right]}\end{array}$ & $\begin{array}{c}\text { Total volume } \\
{\left[\mathrm{mm}^{3}\right]}\end{array}$ & $\begin{array}{c}\text { Porosity } \\
{[\%]}\end{array}$ & DA \\
\hline 7.0 & 58.5 & 88.0 & 0.163 \\
\hline \multicolumn{3}{|c}{ Thickness of walls [mm] } \\
\hline Mean & St. dev. & Max. \\
\hline 0.030 & 0.019 & 0.156 \\
\hline resolution: $3.5 \mu \mathrm{m}$ & \multicolumn{3}{|c}{ sizes of sample: } \\
& $3.22 \times 3.81 \times 4.76 \mathrm{~mm}$
\end{tabular}

TABLE II

Magnetization parameters of magnetic sponge and magnetite nanopowder.

\begin{tabular}{c|c|c|c|c}
\hline \hline Sample & $H_{\mathrm{c}}[\mathrm{Oe}]$ & $M_{\mathrm{r}}[\mathrm{emu} / \mathrm{g}]$ & $M_{\mathrm{s}}[\mathrm{emu} / \mathrm{g}]$ & $M_{\mathrm{r}} / M_{\mathrm{s}}$ \\
\hline $\begin{array}{c}\text { nanopowder } \\
\text { magnetic } \\
\text { sponge }\end{array}$ & $94 \pm 2$ & $9.1 \pm 0.1$ & $76.2 \pm 1.3$ & 0.12 \\
& $87 \pm 2$ & $1.16 \pm 0.02$ & $9.09 \pm 0.22$ & 0.13
\end{tabular}

lar, isotropic, and open porosity. Evaluations performed with the $\mu$-CAT method yielded results directly regarding the structure's parameters, such as total porosity, thickness of the walls, and the microstructure's isotropy. A majority of these parameters is collected in Table I. The thickness of the walls between the pores is proven to be roughly homogeneous in the entire volume of the tested materials, but their surface is not smooth as it is characterised by a diversified topography.

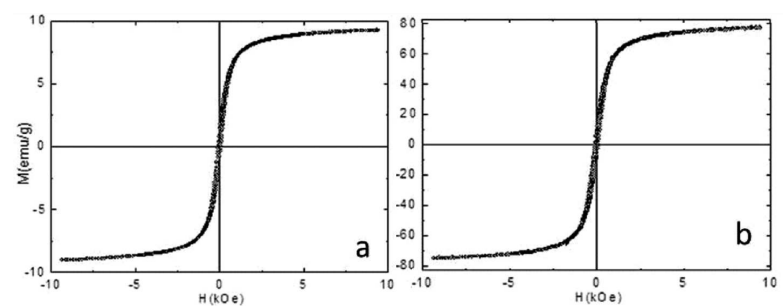

Fig. 5. The magnetic hysteresis at room temperature $(300 \mathrm{~K})$ for magnetic sponge (a) and magnetite nanopowder (b).

Figure 5 shows the magnetic hysteresis of the prepared materials and magnetite nanopowder. The magnetic parameters, including saturation magnetisation $M_{\mathrm{s}}$, coercion $H_{\mathrm{c}}$, and remanence magnetisation $M_{\mathrm{r}}$ are presented in Table II. The coercion fields for both nanoaddition and magnetic sponge are very similar and exhibit a small hysteresis loop. Furthermore, similar values of coercions indicate that both samples have the same ferromagnetic and multi-domain character. In these types of structures the most important influences are connected with mutual, long-range dipole-dipole interactions. Saturation magnetisation and remanence magnetisation strictly depend on the density of the particles' agglomerates. This relation is directly proportional - the saturation magnetisation and remanence magnetisation increase if the particles are located close to each other. On the other hand, the mentioned parameters tend to decrease when particles are well dispersed in the polymer matrix. The distances between various magnetic agglomerates are high enough to prevent mutual influences.

\section{Conclusion}

During these studies a magnetic polymer sponge was successfully fabricated. The preparation method allowed the achievement of both very high porosity and dispersion of the magnetic particles in the volume of polymer 
matrix, which was confirmed by $\mu$-CAT and SEM evaluations, and magnetic measurements. Furthermore, porosity and shape of huge pores may be easily controlled by using different amounts and a various size distribution of the porogen. Lack of sodium chloride crystals in the magnetic sponge confirms a high level of porogen backwash from the polymer matrix. Additionally, it is proven that the presence of the polymer matrix does not change the magnetic properties of the original nanopowder; both the magnetite nanoadditive and the prepared magnetic sponge have a ferromagnetic, multi-domain character.

\section{Acknowledgments}

This work has been supported by the statutory research - 11.11.160.256.

\section{References}

[1] N. Bock, A. Riminucci, C. Dionigi, A. Russo, A. Tampieri, E. Landi, V.A. Goranov, M. Marcacci, V. Dediu, Acta Biomater. 6, 786 (2010).

[2] A.S.P. Lin, T.H. Barrows, S.H. Cartmell, R.E. Guldberg, Biomaterials 24, 481 (2003).

[3] Y. Wan, J. Dai, Q. Zhang, Y. Xiao, M. Lag, Appl. Surf. Sci. 256, 6107 (2012).

[4] H. Wang, Y. Li, Y. Zuo, J. Li, S. Ma, L. Cheng, Biomaterials 24, 3338 (2007).

[5] H. Cao, N. Kuboyama, Bone 46, 386 (2010).

[6] Q. Wu, X. Zhang, B. Wu, W. Huang, Mater. Lett. 92, 274 (2013).

[7] L. Pang, Y. Hu, Y. Yan, L. Liu, Z. Xiong, Y. Wei, J. Bai, Surf. Coat. Technol. 201, 9549 (2007).

[8] V. Canillo, F. Chiellini, P. Fabbri, A. Sola, Compos. Struct. 92, 1823 (2010).
[9] A.M. Deliormanli, M.N. Rahaman, J. Eur. Ceram. Soc. 32, 3637 (2012).

[10] Q. Fu, E. Saiz, A.P. Tomsia, Acta Biomater. 7, 3547 (2011).

[11] S. Chandra, K.C. Barick, D. Bahadur, Adv. Drug Deliver. Rev. 63, 1267 (2011).

[12] A.A.M. Elsherbini, M. Saber, M. Aggag, A. ElShahawy, H.A.A. Shokier, Mag. Reson. Imaging 29, 272 (2011).

[13] A. Figuerola, R. Di Corato, L. Manna, T. Pellegrino, Pharmacol. Res. 62, 126 (2010).

[14] J.O. Mangual, M.O. Avilés, A.D. Ebner, J.A. Ritter, J. Magn. Magn. Mater. 323, 1903 (2011).

[15] H. Kempe, M. Kempe, Biomaterials 31, 9499 (2010).

[16] J. Meng. B. Xiao, Y. Zhang, J. Liu, H. Xue, J. Lei, H. Kong, Y. Huang, Z. Jin, N. Gu, H. Xu, Sci. Rep. 3, 2655 (2013).

[17] S. Panseri, C. Cunha, T. D'Alessandro, M. Sandri, A. Russo, G. Giavaersi, M. Marcacci, C.T. Hung, A. Tampieri, Plos ONE 7, e38710 (2012).

[18] C. Liu, Z. Xia, J.T. Czernuszka, Chem. Eng. Res. Des. 85, 1051 (2007).

[19] H.J. Chung, T.G. Park, Adv. Drug Deliver. Rev 59, 249 (2007)

[20] S.-H. Hu, T.-Y. Liu, C.-H. Tsai, S.-Y. Chen, J. Magn Magn. Mater. 310, 2871 (2007).

[21] S.B. Lee, Y.H. Kim, M.S. Chong, S.H. Hong, Y.M. Lee, Biomaterials 26, 1961 (2006).

[22] D. Sin, X. Miao, G. Liu, F. Wei, G. Chadwick, C. Yan, T. Friis, Mater. Sci. Eng. C 30, 78 (2010).

[23] Q. Hou, D.W. Grijpma, J. Feijen, Biomaterials 24, 1937 (2003). 